Pierre-Alain Duc, Jonathan Braine and Elias Brinks, eds.

\title{
Recycling of Ghost Galaxies: the Origin of giant HI Ring around NGC 1533
}

\author{
Kenji Bekki, Warrick J. Couch \\ School of physics, University of New Southwales, Sydney, NSW, 2052, \\ Australia \\ Emma Ryan-Weber, Rachel Webster \\ School of Physics, University of Melbourne, VIC 3010, Australia
}

\begin{abstract}
We propose that the giant HI ring recently discovered by HIPASS for S0 galaxy NGC 1533 is formed by unequal-mass merging between gas-rich LSB (low surface brightness: "ghost") galaxies and HSB disks. The NGC 1533 progenitor HSB spiral is transformed into a barred S0 during merging and the outer HI gas disk of the LSB is transformed into the giant HI ring. We also discuss two different possibilities for the origin of isolated star-forming regions ("ELdot" objects) in the giant gas ring.
\end{abstract}

\section{Ring formation from tidal disruption of LSBs}

Recent observational results of NGC $1533 \mathrm{HI}$ rings and numerical models for this objects are described in detail by Ryan-Weber et al. (2002) and by Bekki et al. (2003, in preparation), respectively. We accordingly summarize briefly the important three results derived in this numerical studies (See Figure 1 for the model details). Firstly, the strong tidal field of NGC 1533's progenitor HSB strips the outer diffuse HI gas disk of the LSB and completely destroys the stellar disk of the LSB. The stripped HI gas forms an incomplete gas ring and the LSB is swallowed by the HSB finally. This outer HI ring formation is different from the merger scenario of polar ring formation proposed by Bekki (1998). Secondly, the HSB forms a bar and loses its spiral arms owing to tidal heating of the interaction, and therefore can be classified as a barred S0 after merging. Thirdly, star formation can proceed in the giant gas ring either by the cloud-cloud collisions within the ring or by strong external gaseous pressure of the hot gas surrounding the HI ring. The proposed merger models thus explain (1) the observed large total mass of the HI ring, (2) structure and kinematics of the HI ring, and (3) the morphology of

\section{References}

Ryan-Weber, E., Webster, R., \& Bekki, K., 2002, preprint (astro-ph/0209321). Bekki, K., 1998, ApJ, 499, 635 


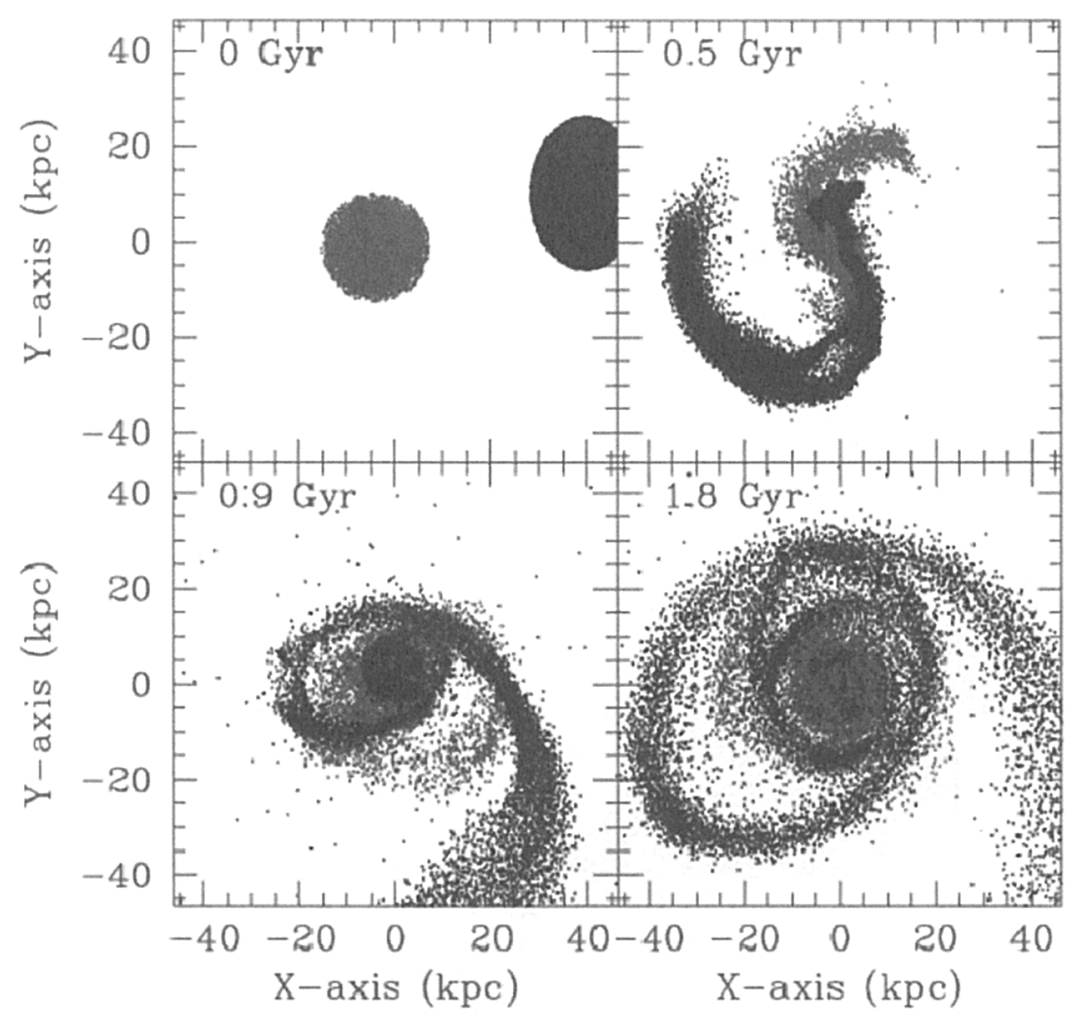

Figure 1. Morphological evolution of the gas of the LSB (dark grey) and the stars of the HSB (light grey) projected onto the $x-y$ plane (see astro-ph/0310358 for a color version of this figure). Both disks are modeled based on the Fall-Efstathiou disk models and the LSB's central surface brightness is 1 mag fainter than that of the HSB. The HI gas disk of the LSB is assumed to extend 2 times optical radius of the LSB. The total HI gas mass of the LSB is assumed to be the same as that of the optical one. The pericenter and the eccentricity of the orbit in this LSB-HSB merger are set to be $0.5 \times R_{\mathrm{d}}$ and 0.8 , respectively, where $R_{\mathrm{d}}$ is the disk size of the HSB. TREESPH code is used and an isothermal equation of state is adopted for the gas with a temperature of $7.3 \times 10^{3} \mathrm{~K}$ (corresponding to a sound speed of $10 \mathrm{~km} \mathrm{~s}^{-1}$ ). Star formation (the Schmidt law) is also included in the SPH simulations. As the two disks merge with each other, only the LSB disk (both gas and stars) is destroyed by the HSB's tidal field to form the outer giant HI gas rings. Since the rings originate from the outer gas disk of the LSB, the rings are composed mostly of gas and the total mass of the ring can be estimated to be more than a few $10^{9} \mathrm{M}_{\odot}$. The shapes of the rings and the velocity field of the HI gas ring reflect the initial LSB's orbit with respect to the HSB. Some local regions along the ring have higher gas density and thus could be the possible formation sites of young stars, which are observed as "ELdots" objects. 